Article

\title{
Investigation of the Anisotropic Thermoelectric Properties of Oriented Polycrystalline SnSe
}

\author{
Yulong Li ${ }^{1,2,3}$, Xun Shi ${ }^{1,2, *}$, Dudi Ren ${ }^{1,2}$, Jikun Chen ${ }^{1,2}$ and Lidong Chen ${ }^{1,2}$
}

1 State Key Laboratory of High Performance Ceramics and Superfine Microstructure, Shanghai Institute of Ceramics, Chinese Academy of Sciences, Shanghai 200050, China; E-Mails: liyulong@student.sic.ac.cn (Y.L.); rendudi@mail.sic.ac.cn (D.R.); artistjackie@163.com (J.C.); cld@mail.sic.ac.cn (L.C.)

2 CAS Key Laboratory of Materials for Energy Conversion, Shanghai Institute of Ceramics, Chinese Academy of Sciences, Shanghai 200050, China

3 University of Chinese Academy of Sciences, Beijing 100049, China

* Author to whom correspondence should be addressed; E-Mail: xshi@mail.sic.ac.cn;

Tel.: +86-21-5241-2803; Fax: +86-21-5241-3122.

Academic Editor: Shi Xue Dou

Received: 31 March 2015 / Accepted: 12 June 2015 / Published: 25 June 2015

\begin{abstract}
Polycrystalline SnSe was synthesized by a melting-annealing-sintering process. $\mathrm{X}$-ray diffraction reveals the sample possesses pure phase and strong orientation along $[h 00]$ direction. The degree of the orientations was estimated and the anisotropic thermoelectric properties are characterized. The polycrystalline sample shows a low electrical conductivity and a positive and large Seebeck coefficient. The low thermal conductivity is also observed in polycrystalline sample, but slightly higher than that of single crystal. The minimum value of thermal conductivity was measured as $0.3 \mathrm{~W} / \mathrm{m} \cdot \mathrm{K}$ at $790 \mathrm{~K}$. With the increase of the orientation factor, both electrical and thermal conductivities decrease, but the thermopowers are unchanged. As a consequence, the $z T$ values remain unchanged in the polycrystalline samples despite the large variation in the degree of orientation.
\end{abstract}

Keywords: thermoelectric; polycrystals; SnSe; anisotropy 


\section{Introduction}

Thermoelectric (TE) materials, which can directly accomplish the interconversion between electric and thermal energy, have been extensively investigated for the application in a power generator to convert waste heat to electrical power or in a refrigerator to pump heat [1-3]. The energy conversion efficiency using TE technology is mainly dominated by materials' figure of merit $(z T)$. So far, lots of advanced thermoelectric materials, such as $\mathrm{Bi}_{2} \mathrm{Te}_{3}, \mathrm{PbTe}$, skutterudites, clathrates, $\mathrm{Cu}_{2} \mathrm{X}(\mathrm{X}=\mathrm{S}, \mathrm{Se})$, $\mathrm{Mg}_{2} \mathrm{Si}$, Half-heusler, have been developed to show the enhanced $z T s$ [4-12], which has attracted increasing interest from industry. A recent report by Zhao et al. shows a high $z T$ of 2.6 at $923 \mathrm{~K}$ in $\mathrm{SnSe}$ single crystal, far above the values of other state-of-the-art TE materials [13]. SnSe is a typical narrow band gap semiconductor and belongs to Pnma space group with orthorhombic structure (lattice parameter $a=11.501, b=4.153$, and $c=4.445 \AA$ ) at room temperature [14,15]. It derives from the NaCl-type structure [16]. The tin atoms are surrounded by highly distorted octahedron of selenium atoms to form a zig-zag structure. Each $\mathrm{Sn}$ atom has six dominant heteropolar bonds, and the Sn-Se bond lengths are 2.74 (1), 2.79 (2), 3.34 (2), and 3.47 (1) $\AA$, where the numbers in the brackets are the number of the Sn-Se bonds in an octahedron [14].When increasing temperature, the Pnma space group changes to a high symmetry orthorhombic $C m c m$ space group (lattice parameter $a=4.310, b=11.705$, and $c=4.318 \AA$ ) around $807 \mathrm{~K}$ as shown in Figure $1[15,17,18]$. In the high-temperature phase, each $\mathrm{Sn}$ (or Se) atom has four neighboring Se (or Sn) atoms with a distance of $3.06 \AA$ in a layered plane.
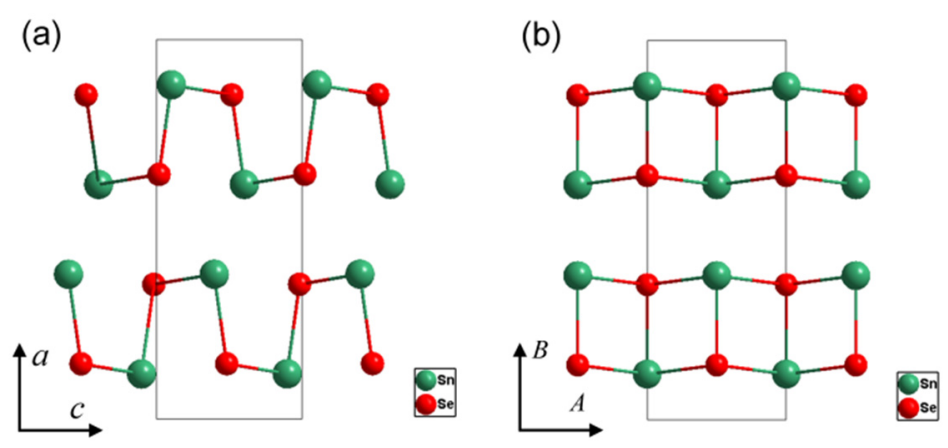

Figure 1. Crystal structures of SnSe at room temperature with Pnma space group (a) and at high temperature with $\mathrm{Cmcm}$ space group (b).

The typical zig-zag structure endows SnSe with anisotropic TE transports in different crystallographic directions. This has been clearly shown in the single crystal samples, where the $b$ and $c$ axes exhibit high electrical and thermal conductivity while the $a$ axis shows low electrical and thermal conductivity, leading to hugely deviated $z T \mathrm{~s}$ along the corresponded $a, b$, and $c$ axes. However, the recent literatures $[19,20]$ show different trends in the polycrystalline SnSe samples where similar $z T$ s are obtained in different oriented directions. In general, the crystallographic orientations in polycrystalline samples are between the two end limits of single crystal sample. Therefore, the thermoelectric properties are expected to between the $b$ (or $c$ ) and $a$ axes. Although the anisotropic transport properties have been reported in polycrystalline SnSe samples [15,19,21], the detailed physical trend and understanding of the anisotropic structure on the electrical and thermal transport as well as thermoelectric figure of merit has not been constructed. 
In this work, we report the investigation of anisotropic thermoelectric properties in oriented polycrystalline SnSe. By collecting and comparing the literature data, the effect of the structure orientation on the electrical and thermal transports will be presented and discussed.

\section{Results and Discussion}

Figure 2a shows the X-ray diffraction patterns of SnSe powders and bulk SnSe polycrystalline sample in the directions parallel to the press $(/ /)$ and perpendicular to the press $(\perp)$. All the peaks are consistent with the standard pattern of SnSe (JCPDS No. 48-1224) with the space group of Pnma, without impurity phases. These results agree well with the energy dispersive spectrometer (EDS) analysis which shows that there is no Sn- or Se-enrichment area throughout the whole bulk sample. The actual composition, SnSe0.99, is obtained for the bulk SnSe polycrystalline sample. Interestingly, high degree of orientation along the $(h 00)$ plane is observed in the SnSe powders. After SPS process, the $(h 00)$ texture diffraction peaks are also maintained in both two directions due to the good crystallinity of the polycrystalline sample along the $(h 00)$ planes. The relative intensities of $(h 00)$ peaks are stronger along the // direction than that along the $\perp$ direction. It implies that the electrical and thermal transport properties in the // direction will be close to that along $a$ axis direction, while the $\perp$ direction will tend towards the $b$ or $c$ axes in the single crystal. The sample morphologies of the freshly fractured surfaces along the $/ /$ and $\perp$ directions observed by SEM are shown in Figure 2b,c, respectively. The obvious layered structures are observed arranging along the // direction. However, the photograph in the $\perp$ direction shows relatively flat planes due to the cleavage of the (100) plane. These findings are consistent with the XRD observations, indicating the oriented structures in polycrystalline SnSe sample.
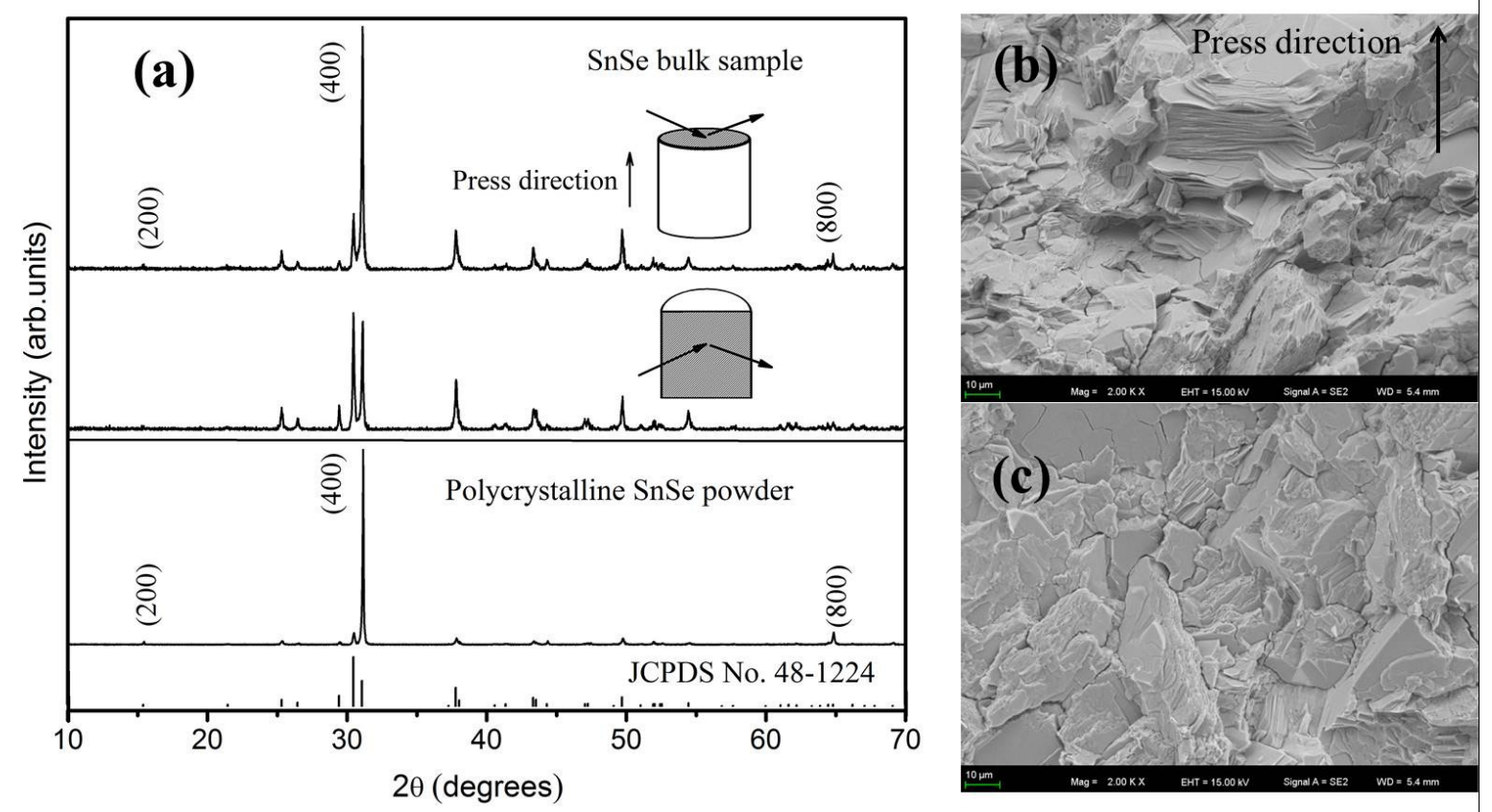

Figure 2. (a) XRD patterns for SnSe powders and bulk sample along different directions; SEM photographs of the freshly fractured surfaces along the directions (b) parallel to the press $(/ /)$ and $(\mathbf{c})$ perpendicular to the press $(\perp)$. 
The degree of the grain orientation in the samples can be described by the orientation factor $(f)$. $f$ is defined as:

$$
\begin{array}{r}
f=\frac{p-p_{0}}{1-p_{0}} \\
p=\frac{\sum_{0}^{2 \theta} I(h 00)}{\sum_{0}^{2 \theta} I(h k l)} \\
p_{0}=\frac{\sum_{0}^{2 \theta} I_{0}(h 00)}{\sum_{0}^{2 \theta} I_{0}(h k l)}
\end{array}
$$

where $I$ and $I_{0}$ are the intensities of XRD reflection peaks for oriented and non-oriented samples, respectively; $p$ and $p_{0}$ are the ratios of the sum of intensities of the $(h 00)$ reflections to that of all $(h k l)$ reflections for oriented and non-oriented samples, respectively [22,23]. The standard diffraction pattern of SnSe (JCPDS No. 48-1224) is used to calculate $p_{0}$. For non-oriented polycrystalline sample, $f$ is 0 , while $f$ is 1 along the $[h 00]$ direction in single crystal. The large $f$ value represents high orientation degree along the [h00] direction in polycrystalline sample. The calculated orientation factors along [h00] directions in the // and $\perp$ directions are 0.41 and 0.13 , respectively (listed in Table 1). Based on the transport data in single crystal, high $f$ means low electrical and thermal conductivities as well as low $z T$. Small $f$ means high electrical and thermal conductivities as well as high $z T$.

Table 1. Orientation factor $f$ along $[h 00]$ directions and room temperature thermal conductivity $\kappa$, electrical conductivity $\sigma$, Seebeck coefficient $S$, Hall carrier concentration $p_{\mathrm{H}}$, Hall mobility $\mu_{\mathrm{H}}$ in polycrystalline SnSe.

\begin{tabular}{ccccccc}
\hline SnSe Direction & $\boldsymbol{f ( h \mathbf { 0 0 } )}$ & $\boldsymbol{\kappa}(\mathbf{W} / \mathbf{m} \cdot \mathbf{K})$ & $\sigma\left(\boldsymbol{\Omega}^{-\mathbf{1}} \mathbf{c m}^{-\mathbf{1}}\right)$ & $\boldsymbol{S}(\boldsymbol{\mu} \mathbf{V} / \mathbf{K})$ & $\boldsymbol{p}_{\mathbf{H}}\left(\mathbf{1 0}^{\mathbf{1 7}} \mathbf{c m}^{-\mathbf{3}}\right)$ & $\boldsymbol{\mu}_{\mathbf{H}}\left(\mathbf{c m}^{\mathbf{2}} / \mathbf{V} \cdot \mathbf{s}\right)$ \\
\hline$/ /$ & 0.41 & 0.89 & 0.91 & 448 & 3.6 & 15.8 \\
$\perp$ & 0.13 & 1.18 & 2.21 & 450 & 1.1 & 123 \\
\hline
\end{tabular}

The electrical properties of the oriented SnSe polycrystalline sample are shown in Figure 3. A drastic element-evaporation is observed when the measurement temperature is higher than $850 \mathrm{~K}$. The surface composition analysis determined by EDS shows that the atomic ratio of $\mathrm{Sn} / \mathrm{Se}$ changes from 1/0.99 to 1/0.60 after the measurements. A dramatic shift on Seebeck coefficient is also observed. As shown in the inset of Figure 3b, the sign of the Seebeck coefficient even changes from positive to negative value above $900 \mathrm{~K}$, further proving that the composition of the sample has been greatly changed. Therefore the other tests are performed from room temperature to $850 \mathrm{~K}$. The reported data of polycrystalline and single crystalline SnSe are also plotted in Figure 3 for comparison $[13,20]$. The electrical conductivity $(\sigma)$, Seebeck coefficient $(S)$, and power factors $\left(S^{2} \sigma\right)$ of our polycrystalline sample are very similar to the literature's data in the whole temperature range. As expected, the electrical conductivity of polycrystalline SnSe along the // direction is small and is close to that along $a$ axis in the single crystal due to the strong orientation along $a$ axis. The electrical conductivity in both the $/ /$ and $\perp$ directions is between those along $a$ axis and that along $b$ (or $c$ ) axis of the single crystal. This is reasonable due to the 
partially oriented structures in polycrystalline sample. The difference in electrical conductivity above phase transition (around $800 \mathrm{~K}$ ) is very weak in these two directions, indicating a small anisotropy in electrical transports in the high temperature phase. This is consistent with the observed crystal structural transformation as shown above, because the thermal dynamics tends to increase the entropy leading to isotropic lattice structures as well as electrical transports.

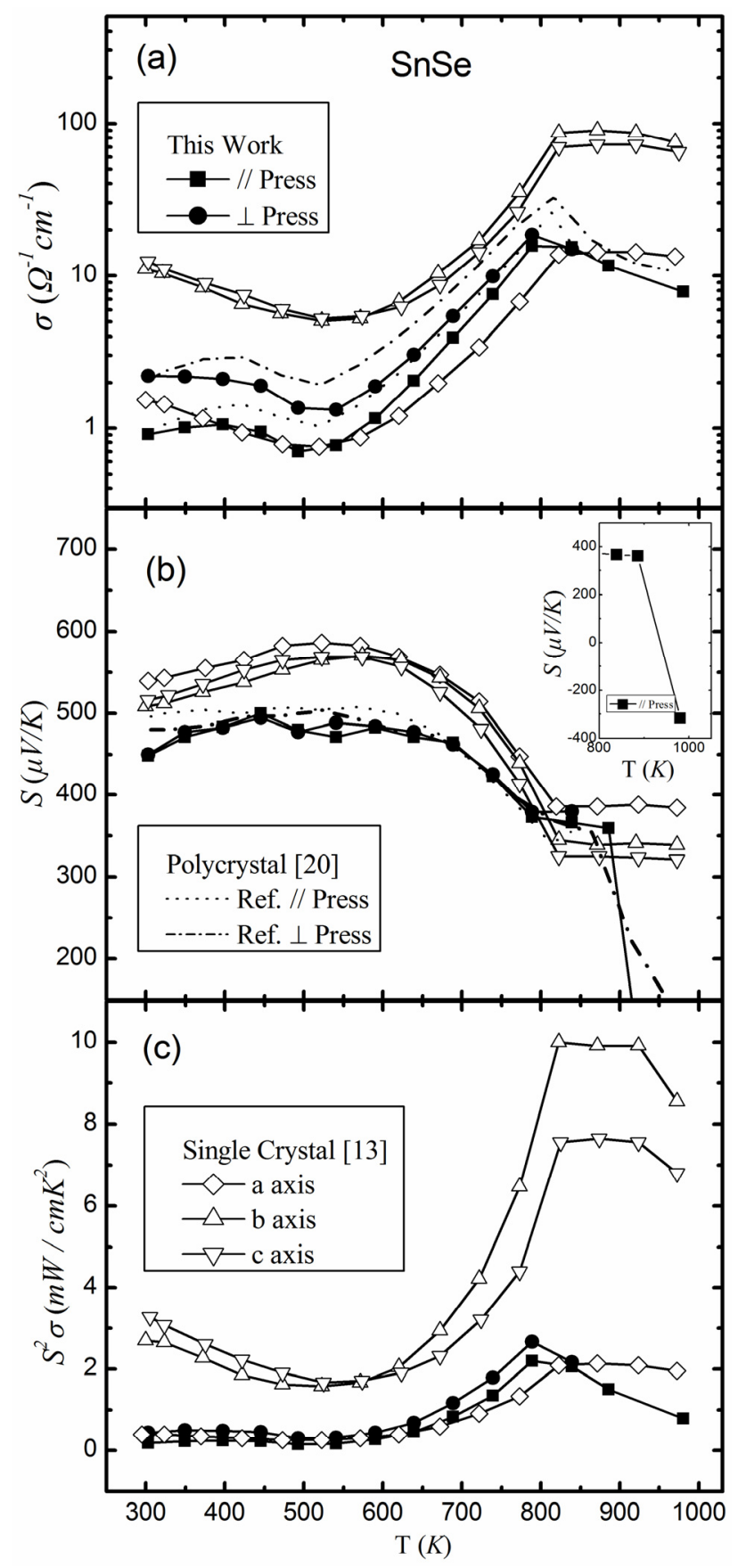

Figure 3. Temperature dependence of (a) electrical conductivity $\sigma$; (b) Seebeck coefficient $S$; and (c) Power Factor $\left(S^{2} \sigma\right)$ for SnSe polycrystalline sample. The inset of (b) is temperature dependence of Seebeck coefficient along the // direction above $800 \mathrm{~K}$.

Similar with single crystal, the difference in Seebeck coefficient of polycrystalline sample along different directions is small, indicating the nearly independence between Seebeck coefficient and 
crystallographic directions in SnSe. This suggests that carrier concentration is the main factor to affect Seebeck coefficient if the effect by grain boundary is ignored. The room-temperature Hall carrier concentrations of our polycrystalline sample with the order of $10^{17} \mathrm{~cm}^{-3}$ along both the $/ /$ and $\perp$ directions (Table 1) are consistent with those in the single crystal [13]. That the electrical conductivities of the polycrystalline samples are between the $a$ axis and $b$ (or $c$ ) axis in the single crystal (Figure 3a) also supports this. Otherwise, the electrical conductivity could be easily tuned in a few orders like that observed in Ag-doped SnSe samples by Chen et al. [19]. This phenomenon has also been observed by S. Sassi et al. [20] in their polycrystalline samples.

The power factors of the polycrystalline sample are shown in Figure 3c. The power factor along the $\perp$ direction is higher than that along the // direction due to the weak orientation in the $\perp$ direction. A maximum power factor of about $2 \mu \mathrm{W} / \mathrm{cm} \cdot \mathrm{K}^{2}$ is obtained in our polycrystalline sample, similar to that in $a$ axis, but much less than those in $b$ or $c$ axis in the single crystal.

Figure 4 shows the temperature dependence of thermal conductivity $(\kappa)$, lattice thermal conductivity $(\kappa \mathrm{L})$ and the dimensionless figure of merit $z T\left(=S^{2} \sigma T / \kappa\right)$ for polycrystalline SnSe. The data from the literatures for polycrystalline and single crystalline SnSe are also plotted in Figure $4[13,19,20]$. SnSe shows relatively low thermal conductivity in comparison with other state-of-the-art TE materials throughout the whole measured temperature range. The lowest thermal conductivity about $0.31 \mathrm{~W} / \mathrm{m} \cdot \mathrm{K}$ is obtained along the // direction at $790 \mathrm{~K}$, and the maximum $z T$ value of 0.56 is obtained along this direction at $790 \mathrm{~K}$.

The lattice thermal conductivity is calculated using the Wiedemann-Franz law with a constant Lorenz number $L_{0}=1.5 \times 10^{-8} \mathrm{~V}^{2} / \mathrm{K}^{2}$, which is the same as that in ref. [13]. Due to the small magnitude of electrical conductivity, the $\kappa_{\mathrm{L}}$ values and its temperature dependence are almost the same as the total thermal conductivity. The lattice thermal conductivity along the // direction is lower than that along the $\perp$ direction. As has been shown in Figure 3a, the polycrystalline sample has small electrical conductivity along the // direction due to the strong electron scattering. Thus the lattice thermal conductivity should be also low along the // direction due to the weak atomic connections and vibrations.

In addition, the polycrystalline SnSe along the // Press and $\perp$ Press directions show similar $z T$ values despite large variance in the degree of orientation. In order to illustrate clearly the effect of orientation on the thermoelectric properties of SnSe, the electrical conductivity $(\sigma)$, Seebeck coefficient $(S)$, power factor $\left(S^{2} \sigma\right)$, thermal conductivity $(\kappa)$, lattice thermal conductivity $(\kappa \mathrm{L})$, and $z T$ value as a function of the orientation factor $f(h 00)$ along [h00] directions are displayed in Figure 5. The data from references [13], [19], and [20] are collected and also plotted in Figure 5. For the single crystal sample in reference [13], the orientation factor along [h00] directions is taken as 0 and 1 along $b / c$ axes and $a$ axis, respectively. For the polycrystalline sample, $f(h 00)$ is 0.083 for the sample of [19] along the $\perp$ direction, 0.034 for the sample of [20] along the $\perp$ direction, and 0.63 for the sample of ref. 20 along the // direction. The carrier and heat transfers are weak along the $[h 00]$ directions, leading to the simultaneous decrease in electrical and thermal conductivities when enhancing $f(h 00)$. The thermopower shows independent trend when shifting the orientations, which gives an essentially unchanged Seebeck coefficient for the polycrystalline SnSe. However, in comparison with the single crystal, the polycrystalline samples show relatively low Seebeck coefficients. This difference is possibly related to the degree of crystallization or 
the effect of grain boundary. Consequently, the $z T$ of the polycrystalline SnSe shows similar values when changing orientation factors. A weak anisotropy of TE figure of merit is observed in the polycrystalline SnSe samples.

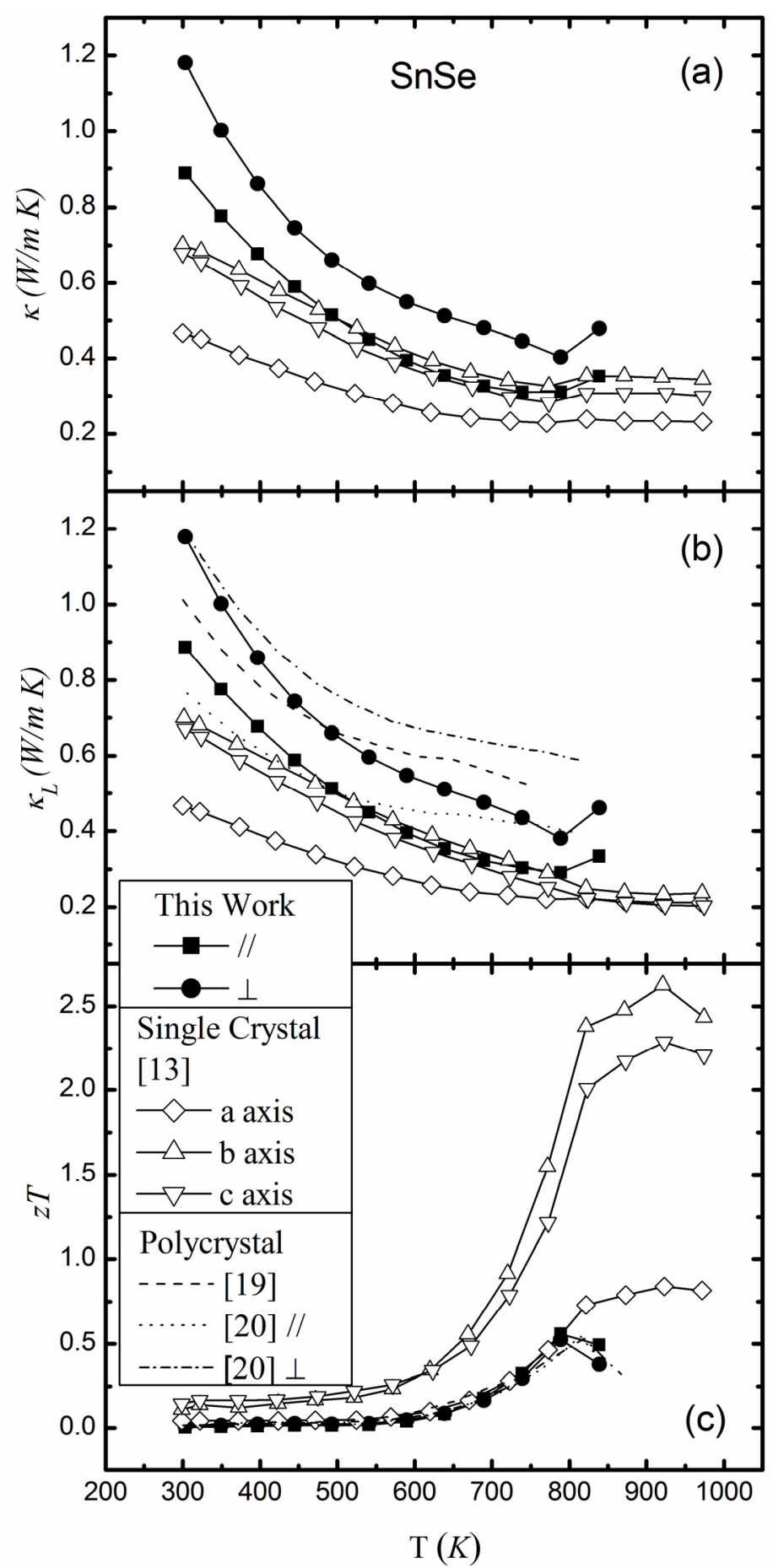

Figure 4. Temperature dependence of (a) thermal conductivity $\kappa$; (b) lattice thermal conductivity $\kappa_{\mathrm{L}}$ and (c) $z T$ for SnSe. The reported data from literatures are plotted for comparison $[13,19,20]$. 

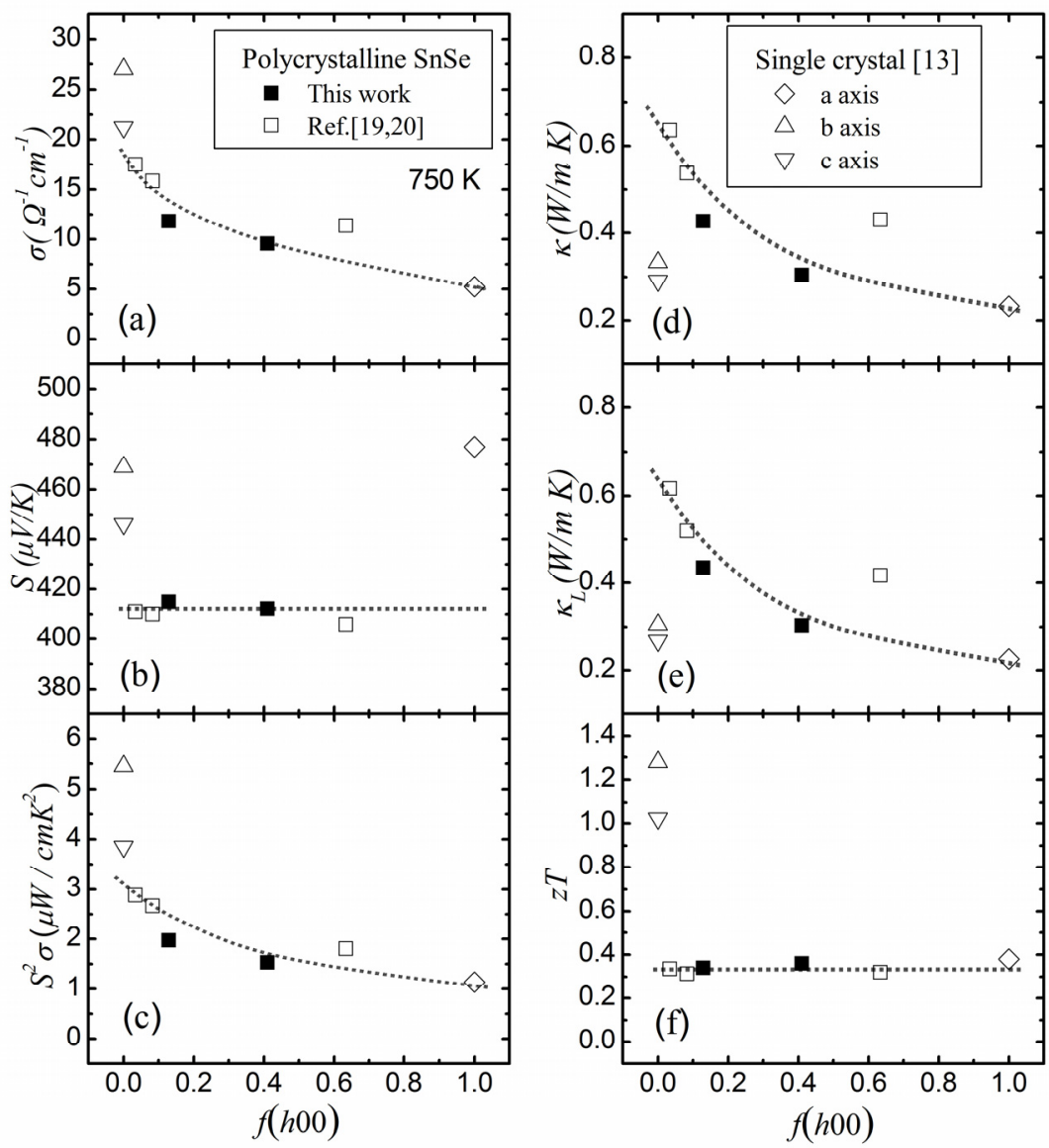

Figure 5. (a) Electrical conductivity $\sigma$; (b) Seebeck coefficient $S$; (c) Power Factor $\left(S^{2} \sigma\right)$; (d) thermal conductivity $\kappa$; (e) lattice thermal conductivity $\kappa \mathrm{L}$; and (f) $z T$ for SnSe as a function of orientation factor along $(h 00)$ directions $f(h 00)$ at $750 \mathrm{~K}[13,19,20]$.

\section{Experimental Section}

Polycrystalline SnSe samples were synthesized by a melting-annealing-sintering process. High purity elements Sn (shot, 99.999\%) and Se (shot, 99.999\%) in the stoichiometric proportion were sealed in evacuated quartz tube. The quartz tube was heated slowly up to $1200 \mathrm{~K}$ and kept at this temperature for $12 \mathrm{~h}$, and then slowly cooled to $900 \mathrm{~K}$ and kept at this temperature for 5 days. The obtained ingot was ground into powder and then sintered by spark plasma sintering at $830-850 \mathrm{~K}$ under a pressure of $60 \mathrm{MPa}$ to obtain densified bulk sample. The density of the bulk sample is $5.92 \mathrm{~g} / \mathrm{cm}^{3}$, which is about $96 \%$ of the theoretical density.

The densified bulk sample was cut into pellets and rectangular bars along the directions perpendicular and parallel to the pressing direction for anisotropic TE property measurement. The phase of the sample was characterized by X-ray diffraction (XRD) analysis (Rint2000, $\mathrm{Cu} \mathrm{K \alpha}$, RIGAKU, Tokyo, Japan). The chemical compositions and morphology of the fractured surfaces of the sample were characterized by energy dispersive spectrometer (EDS, OXFORD, Oxford, England, UK) and Scanning Electron Microscope (SEM, SUPRA 55, ZEISS, Oberkochen, Germany), respectively. The actual compositions were obtained from the average composition of ten different locations on the sample. The measurements of electrical conductivity and Seebeck coefficient were performed in the ZEM-3 system (ULVAC-RIKO, 
Chigasaki, Japan). The thermal diffusivity $(\lambda)$ was measured using the laser flash method in flowing argon atmosphere (LFA 457, NETZSCH, Selb, Germany). The thermal conductivity was calculated by $\kappa=\lambda C_{\mathrm{p}} \rho$, where the specific heat capacity $\left(C_{\mathrm{p}}\right)$ was taken from ref. [13] and the density $(\rho)$ was measured using Archimedes method. The measurements of Hall coefficient $R_{\mathrm{H}}$, was carried out in the Physical Property Measurement System (QUANTUM, San Diego, CA, USA).

\section{Conclusions}

Polycrystalline SnSe was prepared through a melting-annealing-sintering process. The X-ray diffraction patterns show a strong orientation along (h00) directions in both the // and the $\perp$ directions. The polycrystalline sample shows similar carrier concentrations in the order of $10^{17} \mathrm{~cm}^{-3}$ and slightly low Seebeck coefficients compared with single crystal. The thermal conductivities of polycrystalline sample are also very low and the highest $z T$ value of 0.56 is obtained at $790 \mathrm{~K}$. Although the degree of orientation along the // direction is larger than that along the $\perp$ direction, the polycrystalline samples show very weak anisotropic TE performance. With the increase of the orientation factor, both electrical and thermal conductivities decrease, but the thermopowers are almost unchanged. As a result, a weak anisotropy of TE figure of merit is observed in the polycrystalline SnSe samples.

\section{Acknowledgments}

This work was supported by National Basic Research Program of China (973-program) under Project No. 2013CB632501, National Natural Science Foundation of China (NSFC) under the No. 11234012, 51222209 , and 51402337.

\section{Author Contributions}

Yulong Li carried out most of the experiment and data analysis work and prepared the first draft of the paper. Pengfei Qiu and Xun Shi provided the study concept/design and revised the manuscript. The analyses of chemical composition and fracture morphology were performed by Dudi Ren. Jikun Chen participated in data analysis and manuscript revision. Lidong Chen had the overall scientific overview of the procedure and also proofread the paper text. All authors have read and approved the final manuscript.

\section{Conflicts of Interest}

The authors declare no conflict of interest.

\section{References}

1. Rowe, D.M. CRC Handbook of Thermoelectrics; CRC Press: Boca Raton, FL, USA; London, UK; New York, NY, USA; Washington, DC, USA, 1995.

2. DiSalvo, F.J. Thermoelectric cooling and power generation. Science 1999, 285, 703-706.

3. Snyder, G.J.; Toberer, E.S. Complex thermoelectric materials. Nat. Mater. 2008, 7, 105-114. 
4. Poudel, B.; Hao, Q.; Ma, Y.; Lan, Y.; Minnich, A.; Yu, B.; Yan, X.; Wang, D.; Muto, A.; Vashaee, D.; et al. High-thermoelectric performance of nanostructured bismuth antimony telluride bulk alloys. Science 2008, 320, 634-638.

5. Biswas, K.; He, J.; Blum, I.D.; Wu, C.; Hogan, T.P.; Seidman, D.N.; Dravid, V.P.; Kanatzidis, M.G. High-performance bulk thermoelectrics with all-scale hierarchical architectures. Nature 2012, 489, 414-418.

6. Shi, X.; Yang, J.; Salvador, J.R.; Chi, M.; Cho, J.Y.; Wang, H.; Bai, S.; Yang, J.; Zhang, W.; Chen, L. Multiple-Filled Skutterudites: High thermoelectric figure of merit through separately optimizing electrical and thermal transports. J. Am. Chem. Soc. 2011, 133, 7837-7846.

7. Pei, Y.Z.; Yang, J.; Chen, L.D.; Zhang, W.; Salvador, J.R.; Yang, J. Improving thermoelectric performance of caged compounds through light-element filling. Appl. Phys. Lett. 2009, 95, 042101:1-042101:3.

8. Shi, X.; Yang, J.; Bai, S.; Yang, J.; Wang, H.; Chi, M.; Salvador, J.R.; Zhang, W.; Chen, L.; Wong-Ng, W. On the design of high-efficiency thermoelectric clathrates through a systematic cross-substitution of framework elements. Adv. Funct. Mater. 2010, 20, 755-763.

9. Liu, H.; Shi, X.; Xu, F.; Zhang, L.; Zhang, W.; Chen, L.; Li, Q.; Uher, C.; Day, T.; Snyder, G.J. Copper ion liquid-like thermoelectrics. Nat. Mater. 2012, 11, 422-425.

10. He, Y.; Day, T.; Zhang, T.; Liu, H.; Shi, X.; Chen, L.; Snyder, G.J. High thermoelectric performance in non-toxic earth-abundant copper sulfide. Adv. Mater. 2014, 26, 3974-3978.

11. Zaitsev, V.K.; Fedorov, M.I.; Gurieva, E.A.; Eremin, I.S.; Konstantinov, P.P.; Samunin, A.Y.; Vedernikov, M.V. Highly effective $\mathrm{Mg}_{2} \mathrm{Si}_{1-\mathrm{x}} \mathrm{Sn}_{\mathrm{x}}$ thermoelectrics. Phys. Rev. B 2006, 74, 045207:1-045207:5.

12. Sakuradaa, S.; Shutoh, N. Effect of Ti substitution on the thermoelectric properties of ( $\mathrm{Zr}, \mathrm{Hf}) \mathrm{NiSn}$ half-Heusler compounds. Appl. Phys. Lett. 2005, 86, 082105:1-082105:4.

13. Zhao, L.; Lo, S.; Zhang, Y.; Sun, H.; Tan, G.; Uher, C.; Wolverton, C.; Dravid, V.P.; Kanatzidis, M.G. Ultralow thermal conductivity and high thermoelectric figure of merit in SnSe crystals. Nature 2014, 508, 373-377.

14. Wiedemeier, H.; Schnering, H.G. Refinement of the structures of GeS, GeSe, SnS and SnSe. Zeitschrift fur Kristallographie 1978, 148, 295-303.

15. Chattopadhyay, T.; Pannetier, J.; Schnering, H.G. Neutron diffraction study of the structural phase transition in SnS and SnSe. J. Phys. Chem. Solids 1986, 47, 879-885.

16. Okazaki, A. The crystal structure of stannous selenide SnSe. J. Phys. Soc. Jap. 1956, 11, 470.

17. Wiedemeier, H.; Csillag, F.J. The thermal expansion and high temperature transformation of $\mathrm{SnS}$ and SnSe. Zeitschrift fur Kristallographie 1979, 149, 17-29.

18. Schnering, H.G.; Wiedemeier, H. The high temperature structure of $\beta$-SnS and $\beta$-SnSe and the B16-to-B33 type $\lambda$-transition path. Zeitschrift fur Kristallographie 1981, 156, 143-150.

19. Chen, C.; Wang, H.; Chen, Y.; Day, T.; Snyder, G.J. Thermoelectric properties of p-type polycrystalline SnSe doped with Ag. J. Mater. Chem. A 2014, 2, 11171-11176.

20. Sassi, S.; Candolfi, C.; Vaney, J.B.; Ohorodniichuk, V.; Masschelein, P.; Dauscher, A.; Lenoir, B. Assessment of the thermoelectric performance of polycrystalline p-type SnSe. Appl. Phys. Lett. 2014, 104, 212105:1-212105:4. 
21. Carrete, J.; Mingo, N.; Curtarolo, S. Low thermal conductivity and triaxial phononic anisotropy of SnSe. Appl. Phys. Lett. 2014, 105, 101907:1-101907:4.

22. Lotgering, F.K. Topotactical reactions with ferromagnetic oxides having hexagonal crystal structures-I. J. Inorg. Nucl. Chem. 1959, 9, 113-123.

23. Shi, X.; Cho, J.Y.; Salvador, J.R.; Yang, J.; Wang, H. Thermoelectric properties of polycrystalline $\mathrm{In}_{4} \mathrm{Se}_{3}$ and $\mathrm{In}_{4} \mathrm{Te}$. Appl. Phys. Lett. 2010, 96, 162108:1-162108:3.

(C) 2015 by the authors; licensee MDPI, Basel, Switzerland. This article is an open access article distributed under the terms and conditions of the Creative Commons Attribution license (http://creativecommons.org/licenses/by/4.0/). 